\title{
Composição florística do Parque Nacional Serra da Capivara, Piaú, Brasil
}

Jesus Rodrigues Lemos ${ }^{1}$

\section{Resumo}

(Composição florística do Parque Nacional Serra da Capivara, Piaú, Brasil) Dos grandes biomas brasileiros, a caatinga é um dos mais desconhecidos do ponto de vista florístico e fitogeográfico. No Piauí, este bioma reveste cerca de $37 \%$ do seu território, apresentando uma única Unidade de Conservação, o Parque Nacional Serra da Capivara. Este trabalho objetivou listar as espécies coletadas na área do Parque. Para isso, foi realizado um levantamento do material coletado na região, depositado no Herbário "Graziela Barroso" da Universidade Federal do Piauí - TEPB/UFPI, acrescidos de material resultante de coletas realizadas em duas unidades geomorfológicas (sedimentar e cristalino) no período de dois anos. Foram registradas um total de 210 espécies distribuídas em 149 gêneros e 62 famílias. As famílias com maior riqueza foram Caesalpiniaceae, Fabaceae, Mimosaceae, Bignoniaceae, Euphorbiaceae e Myrtaceae com $46 \%$ das espécies. Verificou-se que a heterogeneidade do ambiente físico influencia na distribuição das espécies nos diferentes biótopos da região. No que se refere à região semi-árida como um todo, observou-se que, com exceção de um pequeno grupo de espécies, há semelhança florística entre a área de estudo com outras áreas de caatinga do semi-árido nordestino, quer sejam instaladas em terrenos sedimentares ou sobre o embasamento cristalino. Palavras-chave: semi-árido, caatinga, flora, Piauí, Serra da Capivara.

\section{Abstract}

(Floristic composition of Serra da Capivara National Park, Piauí, Brazil) Of the great Brazilian biomes, the caatinga is one of the most poorly known from the floristic and phytogeografic point of view. In Piaui state, this biome covers about $37 \%$ of its territory, with only one conservation unit, the Serra da Capivara National Park. This work aims at listing the species collected in the Park area. With that objective, a survey was made of the material collected in the area deposited in the Herbarium "Graziela Barroso" of the Universidade Federal do Piauí-TEPB/UFPI, in adittion to samples resultant from collection in two geomorphologic units (sedimentary lands and crystalline soil) during a two year period. It was registered a total of 210 species distributed in 149 genus and 62 families. The families with greater diversity were Caesalpiniaceae, Fabaceae, Mimosaceae, Bignoniaceae, Euphorbiaceae and Myrtaceae totalling $46 \%$ of the species. It was verified that the heterogeneity of the physical environment influences in the distribution of species in the different biotypes of the area. As far as the semi-arid region as a whole is concerned, it was observed that, except for a small group of species, there is floristic similarity between the study area with other areas of caatinga in Brazil's Northeastern native semi-arid, whether installed in sedimentary lands or on crystalline soil.

Key-words: semi-arid, caatinga, flora, Piauí state, Serra da Capivara.

\section{INTRODUÇ̃̃o}

Com base na isoieta modal de $800 \mathrm{~mm}^{-a n o^{-1}}$ e em critérios de natureza geo-ambiental, Souza et al. (1994) afirmaram que a área do semi-árido é de $788.064 \mathrm{~km}^{2}$, onde a precipitação média varia de 400 a $800 \mathrm{~mm}^{2}$.ano $\mathrm{s}^{-1}$ e que além da vegetação de caatinga, ocorrem tipos vegetacionais transicionais em associação com outras formações que ocorrem na Região Nordeste. A caatinga constitui a feição dominante na região semiárida (Andrade-Lima 1960;Fernandes \& Bezerra 1990; Rizzini 1997), apresentando variações florísticas e fisionômicas (Andrade-Lima 1981).
O estado do Piauí ocupa uma posição marginal em relação ao conjunto do Nordeste. Sua quase totalidade e metade do Maranhão formam um conjunto independente denominado "Meio Norte" ou "Nordeste Ocidental", marcando ecologicamente uma zona de transição entre o domínio amazônicoe o semi-áridonordestino. Adistribuição das formações vegetais nestas áreas de transição está ligada, principalmente a fatores climáticos, embora os fatores geomorfológicos e edáficos se tornam determinantes (Emperaire 1989). A influência desses fatores pode ser observada, por exemplo, na região do Parque Nacional Serra

Artigo recebido em 10/2002. Aceito para publicação em 06/2004.

${ }^{1}$ Biólogo, Doutorando em Botânica-USP. Rua Francisco Lucas da Silva, 171 Padre Ibiapina, 62020-701 - Sobral, Ceará, Brasil.jesuslemos@zipmail.com.br 
da Capivara, sudeste do Piauí, onde ocorrem dois conjuntos florísticos e fisionômicos: a formação de caatinga das bacias sedimentares e de terrenos pré-cambrianos (Emperaire 1989).

Até o momento, poucos trabalhos com estudos florísticos, englobando estas duas unidades geomorfológicas foram realizados na região, constando apenas os trabalhos de Emperaire(1984, 1989, 1991), que forneceram registros de algumas espécies vegetais. Com a tentativa de realizar uma complementação destes dados, enriquecendo informações acerca da flora vascular na área do Parque Nacional Serra da Capivara, foi realizado o levantamento florístico na referida área, a partir de exsicatas previamente depositadas no acervo do Herbário "Graziela Barroso", da Universidade Federal do Piauí - TEPB/UFPI, acrescido de material resultante de coletas realizadas na região. Este trabalho pretende, assim, contribuir para um melhor conhecimento da flora da caatinga do Parque, do estado e, conseqüentemente, do semi-árido nordestino.

\section{Material e Métodos}

Área de estudo-O Parque Nacional Serra da Capivara localiza-se no sudeste do estado do Piauí, ocupando áreas dos municípios de São Raimundo Nonato, São João do Piauí, Coronel José Dias e Canto do Buriti (08'26'50' e 08 54'23' S e 42 19'47" e 42\%45'51'O), com a dimensão de 129.953 ha (FUMDHAM 1998). A região do Parque é fronteira entre duas grandes formações geológicas e duas grandes unidades da paisagem do nordeste brasileiro, a Depressão do Médio São Francisco, do embasamento cristalino e a Bacia do Piauí-Maranhão, de sedimentos arenosos (Pellerin 1979, 1991).

A Serra da Capivara forma o limite sul da bacia sedimentar do rio Parnaíba. Apresenta-se como uma chapada com 500 a 600 m de altitude, interrompida por alguns vales. O planalto é formado por camadas areníticas quase horizontais sobre as quais se assentam formações de latossolos areno-argilosos vermelho-amarelado. Nos terrenos pré-cambrianos da depressão, a paisagem é menos acidentada, tratando-se de uma sucessão de pequenos tabuleiros entalhados no sedi- mento. Os solos são também latossolos arenoargilosos vermelhos, porém mais férteis que na zona da bacia sedimentar (Emperaire 1984).

De acordo com Emperaire $(1984,1989)$, a região do Parque situa-se no domínio da caatinga, apresentando tipos de vegetação bem diversificados, com variações fisionômicas que vão desde formações arbóreas altas até formações arbustivas abertas. A autora complementa ainda que essa diversidade de aspectos está principalmente ligada às condições morfo-estruturais.

A precipitação e déficit hídricos médios anuais são de $687,8 \mathrm{~mm}$ e $650 \mathrm{~mm}$ e a temperatura média anual em torno de $26^{\circ} \mathrm{C}$, com chuvas estendendo-se de outubro a maio (Pellerin 1991; Lemos \& Rodal 2002).

Coleta de dados e identificação de material botânico - Foram levantados no acervo do herbário TEPB o material coletado na área do Parque e depositado neste herbário, acrescido de material resultante de coletas realizadas nas duas unidades geomorfológicas (terrenos sedimentares e cristalinos) no período de Setembro/1996 a Dezembro/1998 e distribuídas nos Herbários PEURF, UFP, IPA, TEPB e na Fundação Museu do Homem Americano (FUMDHAM) em São Raimundo Nonato-Piauí. A identificação do material foi realizada utilizando-se literatura especializada e/ou por comparação com exsicatas. A informação referente ao hábito das espécies foi extraída da ficha do herbário e para as novas coletas seguiuse Font-Quer (1977). O sistema de classificação adotado foi o proposto por Cronquist (1981).

\section{Resultados e Discussão}

A Tabela 1 lista 210 taxa distribuídos em 149 gêneros e 62 famílias, dos quais 187 (89\%) identificados no nível de espécie e 23 (11\%) no nível de gênero.

Das famílias levantadas, Caesalpiniaceae (32 spp.), Fabaceae (18 spp.), Mimosaceae (14 spp.), Bignoniaceae (13 spp.), Euphorbiaceae (10 spp.) e Myrtaceae (10 spp.) responderam por $46 \%$ das espécies registradas, enquanto $45 \%$ das famílias apresentaram apenas uma espécie. 
Emperaire $(1984,1989,1991)$ ressalta que a heterogeneidade do ambiente físico influencia na distribuição dos taxa nos diferentes ambientes presentes na região do Parque, o que pôde ser também verificado através da análise realizada neste trabalho.

Das 210 espécies registradas neste levantamento, somente 28 ocorrem tanto em áreas de terrenos sedimentares como em áreas do embasamento cristalino: Spondias tuberosa, Arrabidaea bahiensis, Tabebuia impetiginosa, Tabebuia spongiosa, Cordia leucocephala, Patagonula bahiensis, Neoglaziovia variegata, Bauhinia cheilantha, Bauhinia flexuosa, Caesalpinia bracteosa, Cenostigma gardnerianum, Diptychandra aurantiaca subsp. epunctata, Poeppigia procera, Senna spectabilis, Capparis flexuosa, Erythroxylum betulaceum, Argythamnia gardneri, Cnidoscolus urens, Croton sonderianus, Cratylia mollis, Strychnos rubiginosa, Pavonia glazioviana, Calliandra depauperata, Piptadenia moniliformis, Eugenia cearensis, Cardiospermum corindum, Helicteres baruensis e Turnera blanchetiana.

Segundo a análise realizada por Emperaire (1991) referenteà distribuição geográfica dealgumas espécies presentes no Parque, Cardiospermum corindum (espécie herbácea ruderal) é pantropical; Senna spectabilis, Capparis flexuosa e Cnidoscolus urens são neotropicais; Tabebuia impetiginosa (espécie semi-decídua), Bauhinia cheilantha e Strychnos rubiginosa elementos do cerrado e da caatinga. As espécies que, pela análise deste trabalho, foram encontradas nos dois ambientes do Parque (terrenos sedimentar e cristalino), foram consideradas pela autora, como elementos exclusivos da caatinga.

Das espécies de Caesalpiniaceae ocorrentes no Parque, Bauhinia cheilantha foi comum a áreas cristalinas e sedimentares (embora na área de estudo tenha sido registrada somente em áreas sedimentares), isto parece indicar que a mesma tem ampla distribuição nos tipos caducifólios do semi-árido nordestino (Rodal 1992; Araújoet al. 1995; Ferraz et al. 1998; Araújo et al. 1998; Lemos \& Rodal 2002; Alcoforado-Filhoet al.2003).
De uma maneira geral, observou-se que as áreas sedimentares como um todo, apresentaram um grande número de espécies desta família.

Das 18 espécies de Fabaceae registradas na área, a maioria foi comum a outras áreas sedimentares, sendo que duas espécies: Dalbergia cf. cearensis e Pterodon abruptus foram citadas em vegetação de caatinga instalada sobre o cristalino, embora constatou-se que as mesmas são comuns em áreas de solos arenosos e profundos.

Das Mimosaceae presentes na região, Acacia langsdorfii foi registrada apenas nas áreas sedimentares, enquanto Piptadenia moniliformis ocorreu desde carrasco a caatinga de sedimentos arenosos e do cristalino, indicando a ampla distribuição desta espécie (Lemos \& Rodal 2002).

Bignoniaceae, com 13 espécies registradas neste levantamento, é pouco freqüente nos levantamentos florísticos em áreas do cristalino (Araújo et al. 1995; Alcoforado-Filho et al. 2003), todavia apresenta-se bem expressiva em levantamentos realizados em áreas sedimentares (Oliveira et al. 1997; Araújo et al. 1998; Lemos \& Rodal 2002).

Euphorbiaceae, família bem representada em levantamentos realizados em áreas sedimentares e do cristalino, foi registrada com 10 espécies na área estudada, tendo sido, boa parte das espécies, encontradas nos dois ambientes.

Myrtaceae, com 10 espécies registradas, corroborou com o número de espécies relativamente alto registrado em áreas sedimentares (Oliveira et al. 1997; Araújo et al. 1998; Rodal et al. 1998; Figueirêdo et al. 2000; Rodal et al. 1999; Lemos \& Rodal 2002).

Segundo Rodal (1992), nos diferentes levantamentos que estudaram o componente lenhoso da caatinga instalada em áreas da depressão sertaneja nordestina, Mimosaceae, Caesalpiniaceae e Euphorbiaceae foram as famílias melhor representadas em número de espécies. Já Myrtaceae e Bignoniaceae são pouco representadas naquelas áreas, sendo mais freqüentes em áreas de vegetação caducifólia e perenifólia das chapadas sedimentares (Oliveira et al. 1997; Rodal et al. 1998; Araújo et al. 1998; Figueirêdo et al. 2000; Rodal et al. 1999; Lemos \& Rodal 2002). 
De acordo com análise de levantamentos florísticos e quantitativos realizados nas chapadas sedimentares do semi-árido nordestino (Emperaire 1989; Oliveira et al. 1997; Araújo et al. 1998; Rodal et al. 1999; Araújo \& Martins 1999; Figueirêdo et al. 2000; Lemos \& Rodal 202 Fabaceae, Caesalpiniaceae, Euphorbiaceae e Mimosaceae foram as famílias que demonstraram maior representatividade, fato que sugere uma certa relação com as áreas do cristalino, o que poderia ser explicado pelo condicionante geral da semi-aridez na região como um todo.

Assim, da análise de similaridade florística realizada nos dois conjuntos geomorfológicos da região do Parque Serra da Capivara, verificou- se que a heterogeneidade do ambiente físico (áreas sedimentares ou terrenos cristalinos) influencia na distribuição dos taxa nos diferentes biótopos da região, visto que algumas espécies foram registradas somente em um dos ambientes.

No que se refere à região semi-árida, baseado em análise dos levantamentos florísticos e quantitativos realizados na Região Nordeste, pode-se concluir que, com exceção de um pequeno grupo de espécies registradas somente na área do Parque, há semelhança florística entre a área de estudo com outras áreas de caatinga do semi-árido nordestino, quer sejam instaladas em terrenos sedi-mentares ou sobre o embasamento cristalino.

Tabela 1 - Lista das famílias e espécies ocorrentes no Parque Nacional Serra da Capivara, Piauí. REF. - No de Herbário TEPB e/ou de coleta do autor (JRL); HÁB. - hábito; N. VULGAR - nome vulgar; HABIT. - hábitat; ARV - árvore; ARB - arbusto; SUB - subarbusto; LIA - liana; ERV - erva; C - chapada (terrenos sedimentares); $\mathrm{T}$-tabuleiros (terrenos cristalinos); $\mathrm{LU}$-lugares úmidos; $\mathrm{RU}$-ruderais.

\begin{tabular}{lllll}
\hline FAMÍLIAJESPÉCIE & REF. HÁB. N.VULGAR & HABIT. \\
\hline
\end{tabular}

\section{ACANTHACEAE}

1. Ruellia asperula (Mart. \& Nees) Lindau

\section{AMARANTHACEAE}

2. Amaranthus sp.

3. Gomphrena sp.

ANACARDIACEAE

4. Astronium fraxinifolium Schott ex Spreng

5. Myracrodruon urundeuva Allemão

6. Spondias tuberosa Arruda

\section{ANNONACEAE}

7. Ephedranthus pisocarpus R. E. Fr.

8. Rollinia aff. leptopetala $\mathrm{R}$. E. Fr.

9. Rollinia leptopetala R. E. Fr.

10. Xylopia cf. laevigata(Mart.) R. E. Fr.

11. Xylopia sericea A. St.-Hil.

\section{APOCYNACEAE}

12. Allamanda puberula A. DC.

13. Aspidosperma cf. multiflorum A. DC.

14. Aspidosperma pyrifolium Mart.

ARACEAE

15. Pistia stratoites L.

3813 ERV Melosa-roxa $\quad \mathrm{T}$

$\begin{array}{llll}332 & \text { ERV } & - & \text { RU } \\ 5436 & \text { ERV } & - & \text { RU }\end{array}$

$\begin{array}{llll}6608 & \text { ARV } & \text { Gonçalo alves } & \text { C } \\ 883 & \text { ARV } & \text { Aroeira } & \text { T } \\ \text { JRL92 } & \text { ARV } & \text { Imbuzeiro } & \text { C, T }\end{array}$

$\begin{array}{llll}\text { JRL64 } & \text { ARV } & \text { Cunduru } & \text { C } \\ 6822 & \text { ARV } & \text { Ata brava } & \text { C } \\ 6828 & \text { ARV } & \text { Ata brava } & \text { C } \\ \text { JRL62 } & & & \\ 6829 & \text { ARV } & \text { Cunduru } & \text { C } \\ 6827 & \text { ARV } & - & \text { C }\end{array}$

$\begin{array}{llll}6820 & \text { ARB } & \text { Pente-de-macaco } & \text { C } \\ \text { JRL73 } & \text { ARB } & \text { Pereiro } & \text { C } \\ 6821 & \text { ARV } & \text { Pereiro } & \text { C }\end{array}$




\begin{tabular}{lllll}
\hline FAMIIIAAESPÉCIE & REF. & HÁB. & N.VULGAR & HABIT. \\
\hline ASTERACEAE & & & & \\
16. Eremanthus martii Baker & 91 & ERV & Chico-Rodrigo & - \\
17. Spilanthes cf. acmella (L.) Murr & 163 & ERV & - & RU \\
18. Vernonia remotiflora Rich. & 164 & ERV & - & LU
\end{tabular}

\section{BIGNONIACEAE}

19. Adenocalymma scabriusculum Mart.

20. Arrabidaea bahiensis (Schauer ex DC.)

$\begin{array}{llll}6798 & \text { LIA } & - & \text { C } \\ 6796 & \text { LIA } & \text { Folha-larga } & \text { C, T }\end{array}$

Sandwith \& Moldenke

21. Arrabidaea crassa Spreng.

22. Arrabidaea dispar Bur ex K. Schum

23. Jacaranda brasiliana Pers.

24. Jacaranda jasminoides (Thumb.) Sandwith

25. Mansoa hirsuta DC.

26. Memora axillaris Bur \& K. Schum

27. Memora cf. involucrata Bur \& K. Schum

28. Pyrostegia sp.

29. Tabebuia impetiginosa (Mart. ex DC.) Standl.

30. Tabebuia serratifolia (Vahl) Nicholson

31. Tabebuia spongiosa Rizzini

6797 LIA -

JRL38 LIA Cipó-mole C

6791 ARB Caroba branca C

6816 ARB Caroba preta C

6794 LIA Cipó-de-alho C

JRL49

JRL111 ARB - C

JRL55 ARB Cipó-de-bandeira C

6785 LIA Dedo-de-moça -

6786 ARV Paud'arco roxo C, T

3819 ARV Pau-d'arco C

\section{BOMBACACEAE}

32. Bombax sp.

$599 \quad$ ARV

\section{BORAGINACEAE}

33. Cordia leucocephala Moric.

34. Cordia piauhiensis Fresen.

JRL91 SUB Moleque-duro $\quad$ C,T

JRL32 ARB Grão-de-galo $\mathrm{C}$

3811 ARB Cabo-de-machado C

152 ARB - $C$

$\begin{array}{lllll}\text { 36. Cordia trichotoma (Vell.) Arrab. \& Stend. } & 152 & \text { ARB } & - & \text { C } \\ \text { 37. Heliotropium tiaridioides Cham. } & 1089 & \text { ERV } & \text { Crista-de-galo } & \text { RU }\end{array}$

38. Patagonula bahiensis Moric.

3816 - $\quad$ - $\quad$ C,T

\section{BROMELIACEAE}

39. Bromelia sp.

40. Encholirium spectabile Mart. ex Schult. \& Schult. f.

6592

ERV

$6589 \quad$ ERV

Macambira

Macambira

6591 ERV Caroá

C

41. Neoglaziovia variegata Mez

C, T

\section{CACTACEAE}

42. Cereus albicaulis (Britton. \& Rose) Luetzelb.

43. Pilosocereus sp.

\begin{tabular}{|c|c|c|c|}
\hline JRL74 & ARB & Rabo-de-raposa & $\mathrm{C}$ \\
\hline 6584 & SUB & Xique-xique & $\mathrm{C}$ \\
\hline 6113 & ARB & Miroró, Mororó & $\mathrm{C}$ \\
\hline JRL31 & & & \\
\hline $\begin{array}{l}300 \\
\text { JRL70 }\end{array}$ & ARB & Miroró & $\mathrm{C}, \mathrm{T}$ \\
\hline 6648 & ARB & Miroró & $\mathrm{C}, \mathrm{T}$ \\
\hline JRL112 & ARB & Miroró & $\mathrm{C}$ \\
\hline 125 & ARV & Pau-de-rato & $\mathrm{C}, \mathrm{T}$ \\
\hline 6164 & ARV & Pau-ferro & $T$ \\
\hline
\end{tabular}

\section{CAESALPINIACEAE}

44. Bauhinia acuruana Moric.

45. Bauhinia cheilantha (Bong.) Steud.

46. Bauhinia flexuosa Moric.

47. Bauhinia pentandra (Bong.) Vog. ex Steud.

48. Caesalpinia bracteosa Tul.

49. Caesalpinia ferrea Mart. ex Tul.

ARV

RU T 


\begin{tabular}{|c|c|c|c|c|}
\hline FAMÍLIA/ESPÉCIE & REF. & HÁB. & N.VULGAR & HABIT. \\
\hline 50. Caesalpinia microphylla Mart. ex Tul. & 872 & ARV & Arranca-estribo & $\mathrm{T}$ \\
\hline 51. Cassia ferruginea (Schrad.) Schrad. ex DC. & JRL98 & ARB & - & $\mathrm{T}$ \\
\hline 52. Cenostigma gardnerianum Tul. & $\begin{array}{l}6165 \\
\text { JRL27 }\end{array}$ & ARV & Canela-de-velho & $\mathrm{C}, \mathrm{T}$ \\
\hline $\begin{array}{l}\text { 53. Chamaecrista aff. brevicalyx (Benth.) } \\
\text { Irwin \& Barneby }\end{array}$ & 6649 & ARV & - & - \\
\hline 54. Chamaecrista desvauxii (Collad.) Killip & 198 & ARV & - & - \\
\hline $\begin{array}{l}\text { 55. Chamaecrista eitenorum (Irwin \& Barneby) } \\
\text { Irwin \& Barneby }\end{array}$ & $\begin{array}{l}6647 \\
\text { JRL29 }\end{array}$ & ARV & Subieiro & $\mathrm{C}$ \\
\hline 56. Chamaecrista serpens $(\mathrm{L}$.$) Greene$ & 6625 & ERV & - & - \\
\hline $\begin{array}{l}\text { 57. Chamaecrista aff. zygophylloides (Taub.) } \\
\text { Irwin \& Barneby }\end{array}$ & 6627 & $\mathrm{ARB}$ & - & - \\
\hline 58. Copaifera coriacea Mart. & 6651 & ARV & - & $\mathrm{C}$ \\
\hline 59. Copaifera langsdorffii Desf. & 6626 & ARV & Pau-d’óleo & $\mathrm{C}$ \\
\hline 60. Dimorphandra gardneriana Tul. & JRL113 & ARV & Fava d'anta & $\mathrm{C}$ \\
\hline 61. Diptychandra epunctata Tul. & 779 & ARV & Bilro & $\mathrm{C}, \mathrm{T}$ \\
\hline 62. Hymenaea aurea Lee \& Lang. & $\begin{array}{l}893 \\
\text { JRL39 }\end{array}$ & ARV & Jatobá & $\mathrm{C}$ \\
\hline 63. Hymenaea courbaril L. & 6141 & ARV & Jatobá-trapuca & $\mathrm{C}$ \\
\hline 64. Hymenaea eriogyne Benth. & 6144 & ARV & Jatobazinho & $\mathrm{C}$ \\
\hline 65. Hymenaea stilbocarpa Hayne & JRL90 & ARV & Jatobá & $\mathrm{C}$ \\
\hline 66. Peltogyne confertiflora (Hayne) Benth. & 6080 & ARV & - & $\mathrm{C}$ \\
\hline 67. Poeppigia procera Presl. & $\begin{array}{l}6167 \\
\text { JRL63 }\end{array}$ & ARV & Caracu & $\mathrm{C}, \mathrm{T}$ \\
\hline 68. Sclerolobium densiflorum Benth. & JRL115 & ARB & - & $\mathrm{C}$ \\
\hline 69. Senna cearensis (Afr. Fern.) Afr. Fern. \& E. Nunes & JRL37 & ARB & Pé-de-bode & $\mathrm{C}$ \\
\hline 70. Senna gardneri (Benth.) Irwin \& Barneby & JRL50 & ARB & - & $\mathrm{C}$ \\
\hline $\begin{array}{l}\text { 71. Senna macranthera var. pudibunda (Benth.) } \\
\text { Irwin \& Barneby }\end{array}$ & JRL114 & ARV & - & $\mathrm{C}$ \\
\hline 72. Senna occidentalis (L.) Link. & 119 & $\mathrm{ARB}$ & - & $\mathrm{C}$ \\
\hline 73. Senna trachypus (Benth.) Irwin \& Barneby & JRL110 & SUB & - & - \\
\hline 74. Senna spectabilis (DC.) Irwin \& Barneby & JRL99 & ARB & Canafístula & $\mathrm{C}, \mathrm{T}$ \\
\hline 75. Senna velutina (Vogel.) Irwin \& Barneby & 530 & ARB & - & \\
\hline \multicolumn{5}{|l|}{ CAPPARACEAE } \\
\hline 76. Capparis flexuosa (L.) L. & JRL89 & ARB & Feijão-de-boi & $\mathrm{C}, \mathrm{T}$ \\
\hline 77. Cleome microcarpa Ule & 5435 & ARB & - & - \\
\hline 78. Cleome spinosa $\mathrm{L}$. & 873 & $\mathrm{ARB}$ & Mussambê & $\mathrm{U}$ \\
\hline \multicolumn{5}{|l|}{ CELASTRACEAE } \\
\hline 79. Maytenus sp. & 66 & ARV & Birro branco & $\mathrm{C}$ \\
\hline \multicolumn{5}{|l|}{ CHRYSOBALANACEAE } \\
\hline 80. Licania sp. & JRL109 & ARV & Oiti & $\mathrm{C}$ \\
\hline \multicolumn{5}{|l|}{ COMBRETACEAE } \\
\hline 81. Combretum sp. & 5413 & ARB & Farinha seca & $\mathrm{C}$ \\
\hline 82. Terminalia sp. & 896 & - & Carvoeiro & $\mathrm{T}$ \\
\hline $\begin{array}{l}\text { CONVOLVULACEAE } \\
\text { 83. Jacquemontia densiflora (Miers) Hallier }\end{array}$ & JRL116 & LIA & Jitirana & $\mathrm{C}$ \\
\hline
\end{tabular}




\section{FAMÍLIA/ESPÉCIE \\ ERYTHROXYLACEAE}

84. Erythroxylum betulaceum Mart. 85. Erythroxylum caatingae Plowman 86. Erythroxylum maracasense Plowman

\section{EUPHORBIACEAE}

87. Argythamnia gardneri Müll. Arg.

88. Cnidoscolus phyllacanthus Pax \& K. Hoffm.

89. Cnidoscolus urens (L.) Arthur

90. Croton adenodontus Müll. Arg.

91. Croton campestris A. St.-Hil.

92. Croton sonderianus Müll. Arg.

93. Croton urticaefolius Lam.

94. Croton zehntneri Pax \& K. Hoffm.

95. Manihot caerulescens Pohl

96. Stillingia trapezoides Ule

\section{FABACEAE}

97. Andira vermifuga Mart.

98. Bocoa mollis (Benth.) Cowan

99. Centrosema virginianum (L.) Benth.

100. Cratylia mollis Mart. ex Benth.

101.Dalbergia cearensis Ducke

102. Dioclea grandiflora Mart. ex Benth.

103. Discolobium hirtum Benth.

104. Galactia jussiaeana Kunth

105. Indigofera suffruticosa Mill.

106.Lonchocarpus sericeus (Poir.) Kunthex DC.

107.Luetzelburgia auriculata (Allemão) Ducke

108. Machaerium sp.

109. Macroptilium martii (Benth.) Maréchal \& Baudet

110. Macroptilium panduratum

(Mart. ex Benth.) Maréchal \& Baudet

111. Platypodium elegans Vogel

112. Pterodon abruptus (Moric.) Benth.

113. Swartzia flaemingii Raddi

114. Zornia gardneriana Moric.

\section{FLACOURTIACEAE}

115. Casearia grandiflora A. St.-Hil.

\section{KRAMERIACEAE}

116. Krameria tomentosa A. St.-Hil.

\section{LAMIACEAE}

117. Hyptis multiflora Pohl ex Benth. 118. Hyptis salzmanni Benth.

\section{REF. \\ HÁB. \\ N.VULGAR}

HABIT.

$\begin{array}{llll}\text { JRL52 } & \text { ARB } & \text { Carqueijo } & \text { C, T } \\ \text { JRL77 } & \text { ARB } & - & \text { C } \\ \text { JRL56 } & \text { ARB } & \text { Rompe-gibão } & \text { C }\end{array}$

$\begin{array}{llll}\text { 174 } & \text { ARB } & - & \text { C, T } \\ \text { JRL129 } & \text { ARV } & \text { Favela } & \text { T } \\ \text { JRL128 } & \text { ARB } & \text { Cansanção } & \text { C, T } \\ \text { JRL51 } & \text { ARB } & \text { Malva peluda } & \text { C } \\ \text { JRL108 } & \text { ARB } & \text { Velame } & - \\ \text { JRL107 } & \text { ARB } & \text { Marmeleiro } & \text { C, T } \\ \text { JRL30 } & \text { ARB } & \text { Mulatinha } & \text { C } \\ \text { JRL117 } & \text { ARB } & - & \text { C } \\ \text { JRL68 } & \text { ARV } & \text { Maniçoba } & \text { T } \\ \text { JRL57 } & \text { ARB } & \text { Burraleiteira } & \text { C }\end{array}$

\begin{tabular}{|c|c|c|c|}
\hline 3812 & ARV & Angelim & $\mathrm{C}$ \\
\hline JRL53 & $\mathrm{ARB}$ & Café-brabo & $\mathrm{C}$ \\
\hline 124 & LIA & - & - \\
\hline 780 & $\mathrm{ARB}$ & Camaratuba & $\mathrm{C}, \mathrm{T}$ \\
\hline JRL48 & & & \\
\hline $\begin{array}{l}107 \\
\text { JRL79 }\end{array}$ & ARV & Violete & $\mathrm{C}$ \\
\hline 6143 & LIA & Mucunã & $\mathrm{C}$ \\
\hline 592 & $\mathrm{ARB}$ & - & - \\
\hline 6154 & LIA & - & - \\
\hline 150 & SUB & Anil & RU \\
\hline 583 & ARV & - & $\mathrm{C}$ \\
\hline 891 & ARV & - & - \\
\hline JRL35 & LIA & - & $\mathrm{C}$ \\
\hline 1101 & ERV & - & - \\
\hline 608 & ERV & - & - \\
\hline $\begin{array}{l}259 \\
\text { JRL28 }\end{array}$ & ARV & - & $\mathrm{C}$ \\
\hline $\begin{array}{l}6616 \\
\text { JRL25 }\end{array}$ & ARV & Cangalheiro & $\mathrm{C}$ \\
\hline JRL40 & ARV & Jacarandá & $\mathrm{C}$ \\
\hline 6156 & ERV & Quebra- tigela & RU \\
\hline 3809 & ARV & Ata brava de serrote & $\mathrm{C}$ \\
\hline 148 & - & Carrapicho & - \\
\hline 71 & SUB & - & - \\
\hline 172 & SUB & - & RU \\
\hline
\end{tabular}




\section{FAMÍLIA/ESPÉCIE}

LOGANIACEAE

119. Strychnos rubiginosa DC.

MALPIGHIACEAE

120. Byrsonima cf. gardneriana A. Juss.

121. Heteropterys discolor A. Juss.

122. Peixotoa jussieuana A. Juss.

\section{MALVACEAE}

123. Herissantia tiubae (K. Schum.) Briz.

124. Pavonia cancellata Cav.

125. Pavonia glazioviana Gürke

126. Sida cordifolia L.

127. Sida glomerata Cav. Diss.

\section{MELASTOMATACEAE}

128. Clidemia hirta (L.) Don.

129. Miconia albicans Triana

\section{MELIACEAE}

130. Trichilia hirta L.

\section{MIMOSACEAE}

131. Acacia langsdorffii Benth.

132. Anadenanthera macrocarpa (Benth.) Brenan

133. Calliandra depauperata Benth.

134. Calliandra dysantha Benth.

135. Calliandra leptopoda Benth.

136. Desmanthus virgatus Willd.

137. Mimosa lepidophora Rizzini

138. Mimosa ophthalmocentra Mart. ex Benth.

139. Mimosa somnians Humb. \& Bonpl. ex Willd.

140. Mimosa verrucosa Benth.

141. Parapiptadenia blanchetii (Benth.)

Vaz \& M. P. de Lima

142. Piptadenia moniliformis Benth.

143. Pithecellobium sp.

144. Plathymenia reticulata Benth.

\section{MOLLUGINACEAE}

145. Mollugo verticillata $\mathrm{L}$.

\section{MORACEAE}

146. Brosimum gaudichaudii Trec.

147. Cecropia peltata L.

148.Ficus sp.

\section{MYRSINACEAE}

149. Cybianthus penduliflorus Mart.
REF. HÁB. N.VULGAR

HABIT.

5423

ARB

C, T

JRL33 ARB Murici

JRL41 ARB

JRL45 LIA

$-$

C

$\mathrm{C}$

C

$\begin{array}{llll}1098 & \text { ARB } & - & \text { T } \\ 430 & \text { ERV } & \text { Jitirana-roxa } & \text { RU } \\ \text { JRL26 } & \text { ARB } & \text { Malva-da-chapada } & \text { C, T } \\ \text { JRL96 } & \text { SUB } & \text { Malva babenta } & \text { RU } \\ 143 & \text { ERV } & \text { Malva-dura } & \text { RU }\end{array}$

6803

3814

$-$

-

C,LU

$\mathrm{C}$

6612 ARB Jitó

C

$\begin{array}{llll}\text { JRL23 } & \text { ARB } & \text { Jurema toiceira } & \text { C } \\ 885 & \text { ARB } & \text { Angico-de-umbigo } & \text { T } \\ 797 & \text { ARB } & \text { Carqueijo } & \text { C, T } \\ \text { JRL97 } & \text { ARB } & - & \text { T } \\ 604 & \text { ARB } & - & - \\ 118 & \text { ARB } & \text { Jureminha-branca } & - \\ \text { JRL43 } & \text { ARV } & \text { Umbigo-de-cabra } & \text { C } \\ 5393 & \text { ARV } & - & - \\ 605 & \text { ARB } & - & - \\ 120 & \text { ARV } & \text { Jurema-lisa } & \text { C } \\ \text { JRL127 } & \text { ARV } & - & \mathrm{C} \\ & & & \\ \text { JRL24 } & \text { ARV } & \text { Angico de bezerro } & \mathrm{C}, \mathrm{T} \\ 911 & \text { ARV } & - & - \\ 122 & \text { ARV } & \text { Candeia } & \mathrm{C}\end{array}$

155

ERV Erva de N. Senhora -

$\begin{array}{llll}6130 & \text { ARB } & \text { Minaré } & - \\ 3810 & \text { ARV } & \text { Imbaúba } & \text { C } \\ \text { JRL88 } & \text { ARV } & \text { Gameleira } & \text { C }\end{array}$


FAMILIA/ESPÉCIE

\section{MYRTACEAE}

150. Campomanesia sp.

151. Eugenia cearensis Berg.

152. Eugenia cf. cearensis Berg.

153. Eugenia diantha Berg.

154. Eugenia cf. flava Berg.

155. Eugenia piauhiensis Berg.

156. Eugenia cf. punicifolia (Humb., Bonpl. \& Kunth.) DC.

157. Myrciacf. acutata Berg.

158. Myrciaria ferruginea Berg.

159. Psidium sp.

NYCTAGINACEAE

160. Guapira laxa (Netto) Furlan

161. Pisonia campestris Netto

OCHNACEAE

162. Ouratea sp.

OLACACEAE

163. Ximenia americana $\mathrm{L}$.

\section{OXALIDACEAE}

164. Oxalis euphorbioides A. St.-Hil.

165. Oxalis sepium A. St.-Hil.

\section{PASSIFLORACEAE}

166. Passiflora foetida $\mathrm{L}$.

PHYTOLACCACEAE

167. Microtea sp.

PIPERACEAE

168. Pipersp.

PLUMBAGINACEAE

169. Plumbago scandens L.

\section{POACEAE}

170. Cynodon dactylon (L.) Pers.

171. Rhynchelytum repens(Willd.) C.E. Hubb.

\section{POLYGALACEAE}

172. Polygala variabilis H. B. K.

\section{POLYGONACEAE}

173. Triplaris tomentosa Willd.

\section{PORTULACACEAE}

174. Portulaca elatior Mart.

REF.

HÁB.

N.VULGAR

HABIT.

JRL66

6091

ARB

Guabiraba

Goiaba braba

C

6095

ARB

6100

JRL71

6085

JRL61

6127

JRL42

JRL106

ARB -

ARB

ARV

ARV

ARV

ARB

JRL72 ARV Farinha seca

C

6097

ARV

Maria-mole

C

$900 \quad$ ARB

JRL58 ARB Ameixa

C

$6087 \quad$ ERV

C,LU

$6090 \quad$ ERV

$\mathrm{C}$

3832 LIA Maracujazinho

$5401 \quad$ ERV

C

$6120 \quad$ ARB

6121 ERV Louco

L

171 ERV Capim-burro

170 ERV

6116

6128

ERV

L 


FAMIIIA/ESPÉCIE

175. Colubrina cordifolia Reissek

176. Ziziphus joazeiro Mart.

\section{RUBIACEAE}

177. Diodia teres Walt.

178. Guettarda angelica Mart. ex Müll. Arg.

179. Tocoyena formosa (Schum \& Schl.) Schum.

\section{RUTACEAE}

180. Pilocarpus jaborandi Holmes

181. Zanthoxylum hamadryadicum Pirani

182. Zanthoxylum stelligerum Tuck.

\section{SAPINDACEAE}

183. Allophylus edulis (A. St.-Hil.) Hadlk.

184. Cardiospermum corindum $\mathrm{L}$.

185. Cardiospermum halicacabum L.

186. Magonia glabrata A. St.-Hil.

187. Talisia esculenta Radlk.

\section{SAPOTACEAE}

188. Pouteria gardneriana (A. DC.) Radlk.

SCROPHULARIACEAE

189. Scoparia dulcis L.

\section{SOLANACEAE}

190. Physalis angulata $\mathrm{L}$.

191. Solanum paniculatum L.

\section{STERCULIACEAE}

192. Helicteres baruensis Jacq.

193. Helicteres heptandra L. B. Sm.

194. Helicteres mollis K. Schum.

195. Helicteris muscosa Mart.

196. Melochia tomentosa $\mathrm{L}$.

197. Waltheria sp.

\section{TILIACEAE}

198. Luehea sp.

TURNERACEAE

199. Piriqueta duarteana Urb.

200. Turnera blanchetiana Urb.

201. Turnera calyptrocarpa Urb.

202. Turnera ulmifolia var. guianensis Aubl.

\section{ULMACEAE}

203. Celtis sp.

204. Trema micrantha Blume
REF. HÁB. N.VULGAR HABIT.

6104 ARB Sabão C

JRL75

JRL87

ARV Juazeiro

C

$\begin{array}{llll}\text { 137 } & \text { ERV } & - & \\ 5405 & \text { ARB } & - & \text { T } \\ \text { 173 } & \text { ARB } & \text { Genipapinho } & \text { C } \\ \text { JRL69 } & & & \\ & & & \\ \text { JRL67 } & \text { ARV } & \text { Jaborandi } & \text { C } \\ \text { JRL54 } & \text { ARV } & \text { Pratudo } & \text { C } \\ \text { 5408 } & \text { ARB } & \text { Laranjinha } & \text { C } \\ \text { JRL47 } & & & \\ & & & \text { C } \\ \text { 3833 } & \text { ARV } & - & \text { C, T } \\ \text { 6645 } & \text { LIA } & \text { Chumbinho } & \text { C } \\ \text { JRL118 } & \text { LIA } & \text { Chumbinho } & \text { C } \\ \text { JRL81 } & \text { ARV } & \text { Tingui } & \text { C } \\ \text { JRL105 } & \text { ARV } & \text { Pitombeira } & \end{array}$

JRL86 ARV

C

145 ERV Vassourinha RU

JRL95 SUB Camapu RU

JRL82 ARB Jurubeba C

$\begin{array}{llll}\text { JRL100 } & \text { ARB } & \text { Guaxumbo } & \text { C,T } \\ \text { JRL124 } & \text { ARB } & \text { Guaxumbo } & \text { C } \\ \text { JRL119 } & \text { ARB } & - & \text { C } \\ \text { 34 } & \text { ARB } & - & \text { C } \\ \text { JRL104 } & \text { SUB } & \text { Mutamba-de-roça } & \text { C } \\ \text { JRL94 } & \text { SUB } & \text { Mutamba } & \text { C,LU }\end{array}$

JRL85 - $\quad-\quad$ C

JRL101 ERV Malva-de-vassoura T

$\begin{array}{llll}\text { JRL123 } & \text { ERV } & \text { C }, T\end{array}$

JRL120 ERV -

JRL103 ERV -

JRL122 ARB Mutamba $\quad$ C




\begin{tabular}{|c|c|c|c|c|}
\hline FAMÍLIA/ESPÉCIE & REF. & HÁB. & N.VULGAR & HABIT. \\
\hline \multicolumn{5}{|l|}{ URTICACEAE } \\
\hline 205. Laportea sp. & JRL126 & ARB & - & $\mathrm{C}$ \\
\hline \multicolumn{5}{|l|}{ VELLOZIACEAE } \\
\hline 206. Nanuza aff. plicata (Mart.) L. B. Sm. \& Ayensu & JRL130 & ERV & Canelinha & $\mathrm{T}$ \\
\hline \multicolumn{5}{|l|}{ VERBENACEAE } \\
\hline 207. Lantana camara L. & JRL93 & ARB & Camará & RU \\
\hline \multicolumn{5}{|l|}{ VISCACEAE } \\
\hline 208. Phoradendron sp. & JRL121 & EP & Enxerto & - \\
\hline \multicolumn{5}{|l|}{ VOCHYSIACEAE } \\
\hline 209. Callisthene microphylla Warm. & JRL84 & ARV & Folha miúda & $\mathrm{C}$ \\
\hline 210.Qualea parviflora Mart. & JRL102 & ARV & Pau-terra & $\mathrm{C}$ \\
\hline
\end{tabular}

\section{Agradecimentos}

À Fundação Museu do Homem AmericanoFUMDHAM, co-gestora do Parque Nacional Serra da Capivara, pelo apoio logístico em campo.

\section{REFERÊNCIAS BIBLIGRÁFICAS}

Alcoforado-Filho, F. G.; Sampaio, E. V. S. B.; Rodal, M. J. N. 2003. Florística e fitossociologia de um remanescente de vegetação caducifólia espinhosa arbórea em Caruaru, Pernambuco. Acta Botanica Brasilica 17 (2): 287-303.

Andrade-Lima, D. de. 1960. Estudos Fitogeográficos de Pernambuco. 2 ed. rev. Arquivos do Instituto de Pesquisas Agronômicas 5:305-341.

. 1981. The caatingas dominium. Revista Brasileira de Botânica 4:149-153.

Araújo, E. L.; Sampaio, E. V. S. B. \& Rodal, M. J. N. 1995. Composição florística e fitossociologia de três áreas de caatinga de Pernambuco. Revista Brasileira de Biologia 55 (4): 595-607.

Araújo, F. S.; Sampaio, E. V. S. B.; Figueiredo, M. A.; Rodal, M. J. N. \& Fernandes, A. G. 1998. Composição florística da vegetação de carrasco, Novo Oriente, CE. Revista Brasileira de Botânica 21 (2): 105-116.

Araújo, F. S. de \& Martins, F. R. 1999. Fisionomia e organização da vegetação do carrasco no planalto da Ibiapaba, estado do Ceará. Acta Botanica Brasilica 13 (1): 1-14.

Cronquist, A. 1981. An integrated system of classification of flowering plants. New York, Columbia University Press. 1262 p.

Emperaire, L. 1984. A Região da Serra da Capivara (Sudeste do Piauí) e sua Vegetação. Brasil Florestal 60: 5-21. 1989. Végetation et gestion des ressources naturelles dans la caatinga du sud-est du Piauí (Brésil). Doctorat d'Etat ès Sciences Naturelles, Université Pierre et Marie Curie. Paris, 378p. 1991. Vegetação e flora. In: IBAMA. Plano de Manejo: Parque Nacional Serra da Capivara. IBAMA. Brasília, Distrito Federal. p. 61-206.

Fernandes, A. \& Bezerra, P. 1990. Estudo fitogeográfico do Brasil. Fortaleza, Stylus Comunicações, 205 p.

Ferraz, E. M. N.; Rodal, M. J. N.; Sampaio, E. V. S. B. \& Pereira, R. de C. A. 1998. Composição florística em trechos de vegetação de caatinga e brejo de altitude na região do vale do Pajeú, Pernambuco. Revista Brasileira de Botânica 21 (1): 7-15. Figueirêdo, L. S.; Rodal, M. J. N., Melo, A. L. 2000. Florística e fitossociologia de uma área de vegetação caducifólia espinhosa no município de Buíque - Pernambuco. Naturalia 25: 205-224. 
Font-Quer, M. P. 1977. Diccionario de botanica. Barcelona, Lábor.

FUMDHAM. 1998. Parque Nacional Serra da Capivara. São Raimundo Nonato/Piauí, Fundação Museu do Homem Americano, 94p.

Lemos, J. R. \& Rodal, M. J. N. 2002. Fitossociologia do componente lenhoso de um trecho da vegetação de caatinga no Parque Nacional Serra da Capivara, Piauí, Brasil. Acta Botanica Brasilica 16 (1): 23-42.

Oliveira, M. E. A.; Sampaio, E. V. S. B.; Castro, A. A. J. F. \& Rodal, M. J. N. 1997. Flora e fitossociologia de uma área de transição carrasco-caatinga de areia em Padre Marcos, Piauí. Naturalia 22: 131-150.

Pellerin, J. 1979. Compte-rendu de mission géomorphologique dans la région de São Raimundo Nonato (sud-est du Piauí). Centre de géomorphologie, CNRS, Caen, $15 \mathrm{p}$.

1991. Aspectos físicos. In: IBAMA. Plano de Manejo: Parque Nacional Serra da Capivara. IBAMA. Brasília, Distrito Federal. p. 11-19.

Rizzini, C. T. 1997. Tratado de fitogeografia do Brasil. 2 ed. Rio de Janeiro, Âmbito Cultural Edições Ltda., 747 p.

Rodal, M. J. N. 1992. Fitossociologia da vegetação arbustivo-arbórea em quatro áreas de caatinga em Pernambuco. Tese de Doutorado, Universidade Estadual de Campinas, Campinas, São Paulo. 224p.

Rodal, M. J. N.; Andrade, K. V. de S.; Sales, M. F. de \& Gomes, A. P. S. 1998. Fitossociologia do componente lenhoso de um refúgio vegetacional no município de Buíque, Pernambuco. Revista Brasileira de Biologia 58 (3): 517-526.

Rodal, M. J. N.; Nascimento, L. M. do \& Melo, A. L. de. 1999. Composição florística de um trecho de vegetação arbustiva caducifólia no município de Ibimirim, PE, Brasil. Acta Botanica Brasilica 13 (1): 15-28.
Souza, M. J. N. de; Martins, M. L. R.; Soares, Z. M. L.; Freitas-Filho, M. R. de; Almeida, M. A. G. de; Pinheiro, F. S. de A.; Sampaio, M. A. B.; Carvalho, G. M. B. S.; Soares, A. M. L.; Gomes, E. C. B. \& Silva, R. A. 1994. Redimensionamento da região semi-árida do Nordeste do Brasil. In: Conferência Nacional e Seminário Latino-Americano da Desertificação. Fundação Esquel do Brasil. Fortaleza, Ceará. 25p. 